\title{
MODELO PARA LA ESTIMACIÓN DE LA CONCENTRACIÓN DE BIOMASA EN UNA INSTALACIÓN REACTOR ANÓXICO- FOTOBIORREACTOR AEROBIO DE ALGAS Y BACTERIAS PARA EL TRATAMIENTO DE AGUAS RESIDUALES DOMÉSTICAS
}

\author{
Irina Bausa ${ }^{\mathrm{a}, \mathrm{b}}$, Raúl Muñoz ${ }^{\mathrm{a}, \mathrm{c}}$, Smaranda Podar ${ }^{\mathrm{a}, \mathrm{b}}$, César de Prada ${ }^{\mathrm{a}, \mathrm{b}}$ \\ ${ }^{a}$ Instituto de Procesos Sostenibles, C/ Dr. Mergelina, s/n, 47011, Valladolid \\ ${ }^{\mathrm{b}}$ Dpto. de Ingeniería de Sistemas y Automática, C/ Real de Burgos, s/n, 47011, Valladolid \\ ${ }^{c}$ Dpto. de Ingeniería Química y Tecnología del Medio Ambiente, C/ Dr. Mergelina, s/n, 47011, Valladolid \\ irina.bausa@uva.es, mutora@iq.uva.es, smaranda@autom.uva.es, prada@autom.uva.es
}

\begin{abstract}
Resumen
En este trabajo se presenta el modelado mecanístico de una configuración reactor anóxicofotobiorreactor aerobio de algas y bacterias con recirculación de biomasa para el tratamiento de aguas residuales domésticas. El modelo desarrollado permite estimar la concentración total de sólidos en suspensión en cada uno de los elementos que conforman la instalación. Con el fin de ajustar el modelo a los datos experimentales, se realiza la estimación de los principales parámetros que influyen sobre la concentración total de biomasa en ambos reactores y el sedimentador. El modelo obtenido se valida mediante simulación, comparando los resultados del mismo con los datos experimentales.
\end{abstract}

Palabras clave: Estimación de parámetros, Modelado, Sedimentación, Simbiosis microalgasbacterias, Tratamiento de aguas residuales.

\section{INTRODUCCIÓN}

En los últimos años, las tecnologías de depuración de aguas residuales basadas en consorcios microalgasbacterias han generado un interés creciente dentro de la comunidad científica. Aunque esta tecnología fue propuesta en los años 60, aún permanecen algunas limitaciones relacionadas con su aplicación a gran escala. Las directivas medioambientales de la Unión Europea en cuanto a los procesos de tratamiento de aguas residuales; la gestión eficiente de los nutrientes; y el tránsito hacia una economía hipocarbónica, eficiente en el uso de los recursos, ecológica y competitiva, han reactivado el interés por estas tecnologías y el desarrollo de un gran número de mejoras y aplicaciones [10].

En los sistemas convencionales de tratamiento de aguas residuales basados en lodos activos, se estima que los costos asociados a la aireación mecánica representan entre el 45-75\% de los costos operacionales totales [7]. Estos costos pueden reducirse empleando sistemas de depuración basados en consorcios microalgas-bacterias, ya que las microalgas constituyen una fuente de oxígeno $\left(\mathrm{O}_{2}\right)$ fotosintético, a la vez que reducen las emisiones de dióxido de carbono $\left(\mathrm{CO}_{2}\right)$ derivadas de la oxidación de la materia orgánica [11]. La capacidad de las microalgas para eliminar simultáneamente carbón $(\mathrm{C})$, nitrógeno $(\mathrm{N})$ y fósforo $(\mathrm{P})$ mediante asimilación mixotrófica representa una ventaja importante en términos de recuperación de nutrientes respecto a la tecnología aerobia de lodos activos o a la de digestión anaerobia [3]. En este sentido, los sistemas de tratamiento de aguas residuales basados en microalgas representan una oportunidad para reemplazar los sistemas convencionales de saneamiento por "industrias productivas" [14]. La biomasa microalgal obtenida en los sistemas de tratamiento de aguas residuales no puede utilizarse directamente en aplicaciones humanas; en cambio, puede ser cosechada para su aplicación en diferentes aspectos: como suplemento o complemento de la alimentación animal, producción de biofertilizantes, bioestimulantes, bioplástico y biodiesel [1], [4].

El número creciente de aplicaciones de tecnologías basadas en microalgas ha fomentado el desarrollo de nuevos modelos para el estudio de los principales procesos, factores y variables que influyen en el crecimiento de las microalgas en diferentes medios de cultivo, incluyendo las aguas residuales [5], [12], [14]. Los modelos matemáticos ofrecen una gran oportunidad para estudiar simultáneamente el efecto de los múltiples factores que afectan a las microalgas y bacterias, permitiendo así la predicción de la producción final de biomasa y contribuyendo a la optimización del diseño del sistema en términos de operación y control [13].

Recientemente, otra de las líneas de investigación que ha generado gran interés es el diseño y mejora de instalaciones que optimizen el rendimiento de la biomasa de algas y garanticen la adecuada 
depuración de las aguas residuales. En este sentido, en [8] se propone una configuración de reactor anóxico-fotobiorreactor aerobio para eliminar el nitrógeno y fósforo del agua residual y mejorar la sedimentación de biomasa microalgal. Esta instalación fue optimizada en [2] con el objetivo de promover la eliminación de nitrógeno vía desnitrificación y el desarrollo de una rápida sedimentación de la población de microalgasbacterias.

El objetivo de este trabajo es modelar, por primera vez, la configuración reactor anóxico-fotobiorreactor aerobio descrita en [2] y determinar los valores óptimos de los parámetros del modelo. El modelado y simulación del proceso se realizan en el entorno de simulación dinámica PROOSIS®.

En este trabajo se consideran como variables de salida del modelo el Total de Sólidos en Suspensión (Total Suspended Solids, TSS) en ambos reactores y el sedimentador, debido a que el valor del mismo a la salida de la planta no debe exceder el límite máximo indicado en la legislación de la Unión Europea para los vertidos procedentes de instalaciones de depuración de aguas residuales urbanas [6]. Por lo tanto, el valor de concentración de TSS en el efluente y el porcentaje de reducción en relación con el caudal de entrada, constituyen indicadores relevantes en el diseño y evaluación de una instalación depuradora. Además, en el fotobiorreactor valores de biomasa alejados del óptimo conllevarían problemas de fotoinhibición o apantallamiento de la luz, con la consiguiente disminución en la oxigenación fotosintética del proceso, que a su vez impacta en los rendimientos de eliminación de materia orgánica y nitrificación.

El trabajo está estructurado en cuatro secciones: a continuación, se realiza la descripción de la planta y de cada uno de los componentes de la instalación. En la Sección 3 se hace referencia al modelado de la instalación. La Sección 4 está dedicada al procedimiento de estimación de parámetros; y por último, en la Sección 5, se presentan los resultados de aplicación del modelo.

\section{DESCRIPCIÓN DE LA PLANTA}

La planta consiste en un proceso de microalgas y bacterias reactor anóxico-fotobiorreactor aerobio con recirculación de biomasa [8]. En [2] se recogen los resultados de los experimentos realizados con el objetivo de estimular la eliminación de nitrógeno del agua residual. Se analizan la influencia del Tiempo de Retención Hidráulico (Hydraulic Retention Time, $H R T$ ), intensidad y régimen de luz y concentración de oxígeno disuelto en el fotobiorreactor. La influencia de estas condiciones de operación sobre la eliminación de carbono y nitrógeno, se recoge en cinco etapas de experimentación.

La instalación experimental consta de un tanque anóxico interconectado con un fotobiorreactor, tal como se representa en el diagrama en bloques de la Figura 1. El fotobiorreactor es un tanque encamisado de vidrio de 3.5 L (AFORA, España) con un volumen de trabajo total de $2.7 \mathrm{~L}$. El fotobiorreactor fue iluminado de manera continua por tiras de luces LED de $4 \times 5$ metros (F30W-12V, España) colocadas de forma circular, las cuales proveen una iluminación de $400 \pm 51 \mu \mathrm{E} / \mathrm{m}^{2} \mathrm{~s}$ en la pared externa del fotobiorreactor. La temperatura y la agitación magnética del fotobiorreactor se mantuvieron constantes a $24 \pm 1{ }^{\circ} \mathrm{C}$ y $300 \mathrm{rpm}$, respectivamente, mientras que el $\mathrm{pH}$ se mantuvo a $7.8 \pm 0.1$ mediante la adición diaria de $0.8 \mathrm{~mL}$ de ácido clorhídrico (37\%) durante las etapas I y II.

El reactor anóxico consiste en un tanque hermético de cloruro de polivinilo de $1 \mathrm{~L}$ con un volumen de trabajo total de $0.9 \mathrm{~L}$, este tanque se mantuvo en la oscuridad y agitado continuamente a $300 \mathrm{rpm}$. El agua residual sintética utilizada en los experimentos fue previamente esterilizada a $121^{\circ} \mathrm{C}$ durante $20 \mathrm{~min}$ y se mantuvo a $7^{\circ} \mathrm{C}$. Con la esterilización del agua residual sintética se evita el crecimiento de microorganismos dentro del tanque de alimentación que degraden la materia orgánica y los nutrientes antes de entrar al sistema de biorreactores. De esta forma, se garantiza que la composición del agua residual se mantenga constante durante todo el tiempo del experimento.

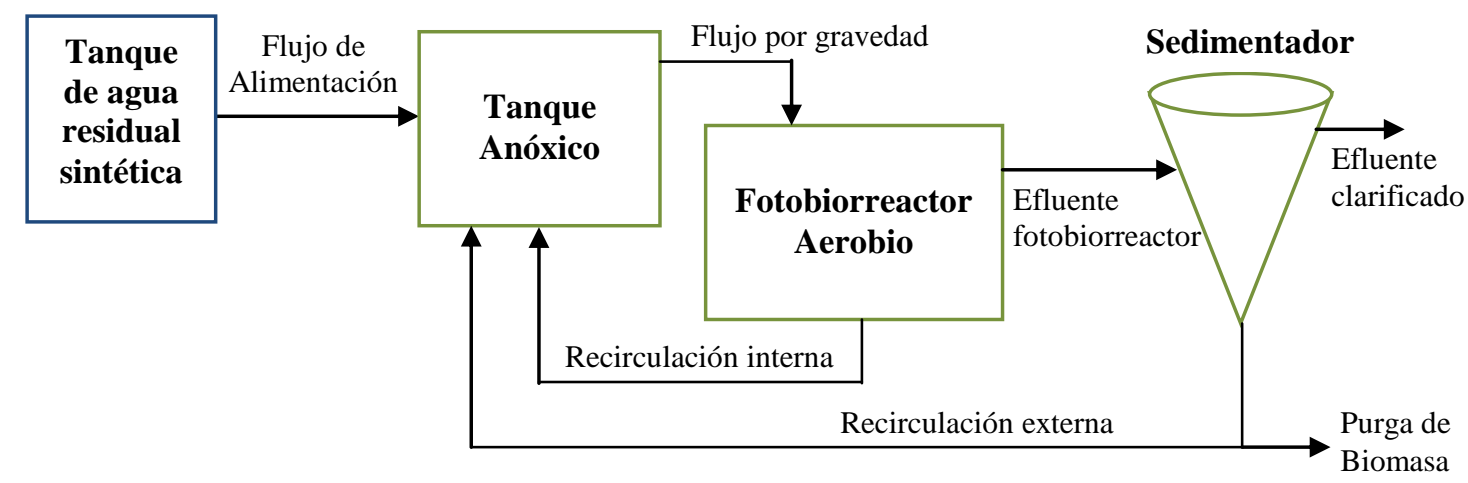

Figura 1: Diagrama en bloques de la configuración reactor anóxico-fotobiorreactor 
El reactor anóxico se alimenta con el agua residual sintética y el fotobiorreactor se alimenta continuamente por gravedad con el flujo de rebose del reactor anóxico (Figura 1). El cultivo de microalgas-bacterias se recirculó de manera continua con un flujo de $3 \mathrm{~L} / \mathrm{d}$ desde el fotobiorreactor al reactor anóxico con el objetivo de suministrar los nitritos $\left(\mathrm{NO}_{2}^{-}\right)$y nitratos $\left(\mathrm{NO}_{3}^{-}\right)$(generados en el fotobiorreactor mediante nitrificación biológica) requeridos para la desnitrificación. La temperatura del tanque anóxico se mantuvo constante a $24 \pm 1^{\circ} \mathrm{C}$. Un cono de Imhoff de $1 \mathrm{~L}$ de volumen interconectado con la salida del fotobiorreactor se utilizó como sedimentador. La biomasa de microalgas-bacterias sedimentada se recirculó desde el fondo del sedimentador hacia el tanque anóxico a $0.5 \mathrm{~L} / \mathrm{d}$ y se purgó tres veces a la semana con el objetivo de controlar el tiempo de retención de lodos (Sludge Retention Time, SRT) del consorcio microalgasbacterias.

Los estudios a escala de planta piloto aportan una idea acerca de las características del efluente que se obtendrá, aunque existen problemas de escala y en general únicamente son útiles como estudios iniciales. A mayor escala podría existir estratificación de la biomasa dentro del fotobiorreactor y el régimen de mezcla no se correspondería con mezcla perfecta. Aun así, estos estudios resultan de gran utilidad para evaluar diferentes condiciones de operación y estrategias de control, pues permiten trabajar en un ambiente controlado.

\subsection{DISEÑO DEL EXPERIMENTO}

La experimentación se diseñó basada en la hipótesis de que los fotobiorreactores de algas y bacterias para el tratamiento de aguas residuales pueden oxidar el amonio $\left(\mathrm{NH}_{4}{ }^{-}\right)$en $\mathrm{NO}_{2}{ }^{-} / \mathrm{NO}_{3}{ }^{-}$, los cuales se pueden eliminar fácilmente mediante el proceso de desnitrificación heterótrofa (utilizando la materia orgánica presente en el agua residual sintética) bajo condiciones anóxicas, mediante la recirculación del cultivo del fotobiorreactor [8]. Se tomaron $100 \mathrm{~mL}$ de muestras líquidas tres veces a la semana del agua residual sintética, reactor anóxico, fotobiorreactor aerobio, purga y efluente clarificado, con el fin de monitorear las concentraciones del Total de Carbono Orgánico (Total Organic Carbon, TOC) disuelto, Carbono Inorgánico (Inorganic Carbon, IC) disuelto, especies de nitrógeno disueltas (nitrógeno total, $\mathrm{N}$ $\mathrm{NH}_{4}^{-}, \mathrm{N}-\mathrm{NO}_{2}^{-}, \mathrm{N}-\mathrm{NO}_{3}^{-}$) y concentración de biomasa, expresada como Total de Sólidos en Suspensión. La Concentración de Oxígeno Disuelto (Dissolved Oxygen Concentration, DOC), la temperatura y el $\mathrm{pH}$ del medio de cultivo en ambos tanques se midieron in situ diariamente.

Los datos obtenidos durante las dos primeras etapas de operación se han utilizado en este trabajo como variables de entradas y salidas, para ajustar el modelo matemático de esta instalación. En la etapa I, el sistema opera a un flujo de agua residual de $0.9 \pm 0.1$ L/d y en la etapa II a $1.7 \pm 0.2 \mathrm{~L} / \mathrm{d}$, manteniéndose en ambas etapas los flujos de recirculación interna y externa en $3 \mathrm{~L} / \mathrm{d}$ y $0.5 \mathrm{~L} / \mathrm{d}$, respectivamente. El desarrollo del modelo matemático permitirá variar las condiciones de operación para predecir cambios en las salidas del proceso, reduciendo significativamente el tiempo dedicado a la experimentación.

\section{MODELO DE LA PLANTA}

Existen diferentes modelos matemáticos que describen las reacciones y procesos para las bacterias en sistemas de tratamiento de agua residual convencionales como el sistema de lodos activos, estos se han desarrollado y validado exitosamente y en la actualidad son ampliamente aceptados y aplicados. Por el contrario, los modelos que describen las complejidades internas en sistemas de tratamiento de aguas basados en microalgasbacterias, se considera que aún están en proceso de desarrollo [13]. Esto se debe a que, en comparación con las tecnologías de tratamiento convencionales, resultan menos conocidas las reacciones y procesos físicos, químicos y bioquímicos que se producen en los sistemas de tratamiento basados en microalgas y bacterias. Los modelos matemáticos ofrecen la oportunidad de estudiar las interacciones microalgasbacterias y aportan valiosas herramientas para el diseño y control, lo cual deriva en un incremento en la eficiencia de los fotobiorreactores. La revisión de varios de estos modelos, permitió tomar como punto de partida para el presente trabajo el modelo BIO_ALGAE [15], debido a que este incluye las interacciones entre microalgas y bacterias, sin presentar una complejidad excesiva.

La viabilidad de la tecnología de tratamiento de aguas residuales utilizando una configuración reactor anóxico-fotobiorreactor aerobio ha sido probada experimentalmente a escala de laboratorio en el Instituto de Procesos Sostenibles de la Universidad de Valladolid [2]. Con esta configuración, además de obtenerse altos valores de eficiencias de eliminación de nitrógeno total (68-79\%), se consiguieron altos valores de eficiencias de eliminación de carbono orgánico total (86-89\%) y carbono inorgánico (57$98 \%$ ). Para la evaluación de esta configuración en [2] se diseñaron cinco etapas, correspondientes a diferentes condiciones de operación, cada etapa con una duración entre 32 y 47 días, necesitándose un total de 186 días para completar el proceso de experimentación.

El modelo BIO_ALGAE, aplicado al sistema descrito en [2], se ha utilizado para describir las relaciones fundamentales que tienen lugar en el reactor anóxico y el fotobiorreactor, mientras que el sedimentador se ha modelado utilizando expresiones de balance de 
masas. El modelado y simulación del proceso se ha realizado en el entorno de simulación dinámica PROOSIS $®$ [9].

El modelo BIO_ALGAE utiliza la nomenclatura de los modelos de la Asociación Internacional del Agua (International Water Association, IWA) y considera 19 componentes (6 particulados y 13 disueltos) implicados como variables en los procesos físicos, químicos y biocinéticos. En [15] se describen estos componentes, el papel que desempeñan en los procesos que tienen lugar en los fotobiorreactores y las interacciones con otros componentes.

\subsection{MODELO DEL REACTOR ANÓXICO}

En el reactor anóxico se han utilizado las expresiones descritas en el modelo BIO_ALGAE [15] para modelar los procesos que tienen lugar en el mismo.

En el caso de las microalgas, sólo se tiene en cuenta el proceso correspondiente a la muerte de las microalgas. En cuanto a la actividad de las bacterias heterótrofas, se consideran los procesos relativos al crecimiento anóxico (en $\mathrm{S}_{\mathrm{NO} 2}$ y $\mathrm{S}_{\mathrm{NO} 3}$ ), la respiración endógena anóxica y la muerte de bacterias heterótrofas. En el caso de las bacterias nitrificantes, en el reactor anóxico sólo se consideran los procesos de muerte de las bacterias oxidantes de amonio y muerte de las bacterias oxidantes de nitritos.

En este reactor, en las ecuaciones diferenciales que describen las variables de estado del sistema, se consideran los términos relativos al transporte de masa de cada componente, teniendo en cuenta los flujos de entrada y salida al reactor. El reactor anóxico, tal como se representa en la Figura 1, recibe flujos de entrada del tanque de agua residual sintética, el fotobiorreactor (recirculación interna) y el sedimentador (recirculación externa).

\subsection{MODELO DEL FOTOBIORREACTOR AEROBIO}

El fotobiorreactor se modela teniendo en cuenta los procesos descritos en el modelo BIO_ALGAE [15]. En las velocidades de crecimiento de estos procesos se tiene en cuenta el efecto de la concentración de nutrientes (limitación o inhibición) y de las condiciones ambientales (luz, temperatura y oxígeno disuelto).

Particularmente, en cuanto a la actividad de las bacterias heterótrofas, se tiene en cuenta el crecimiento aerobio de las mismas (en $\mathrm{S}_{\mathrm{NH} 4}$ y $\mathrm{S}_{\mathrm{NO} 3}$ ), el proceso de respiración endógena aerobia y la muerte de las bacterias heterótrofas.

En los procesos que ocurren en el fotobiorreactor, se han considerado, además, los términos relativos al transporte de masa de cada componente, teniendo en cuenta el flujo de entrada del reactor anóxico y los flujos de salida hacia el reactor anóxico y hacia el sedimentador.

\subsection{MODELO DEL SEDIMENTADOR}

El sedimentador se modela tomando como base el modelo de Takács et al. [16]. En este modelo, se supone que en el sedimentador no tienen lugar reacciones biológicas. El sedimentador se considera formado por un conjunto de capas, de manera que el flujo de sólidos debido a la gravedad depende de la concentración de fangos en la misma. Las partículas que entran se distribuyen de manera uniforme e instantánea a través de la capa de entrada y las ecuaciones del modelo sólo consideran el flujo en sentido vertical. El flujo de sólidos en cada capa se denomina $J$ y depende de la concentración de sólidos en la capa $(X)$ y de la velocidad $(v)$, tal como se refiere en la expresión (1).

$$
J=v(X) X
$$

En la descripción de las ecuaciones de estado del modelo de Takács [16], se establecen cinco tipos de capas, dependiendo de su posición respecto a la capa de alimentación: la capa superior, las capas superiores a la de alimentación, la capa de alimentación, las capas inferiores a la de alimentación y la capa inferior.

En el modelado de sedimentadores, es fundamental el estudio de la velocidad de sedimentación (2), esta se describe por el modelo de doble exponencial propuesto en [16], este modelo es válido tanto para la zona de sedimentación zonal, como para la zona de clarificación.

$$
V_{s}=V_{0}\left(e^{-r_{h}\left(X-X_{\min }\right)}-e^{-r_{p}\left(X-X_{\min }\right)}\right) \quad 0 \leq V_{s} \leq V_{0}^{\prime}
$$

donde:

$\mathrm{X}_{\min }$ : Concentración de sólidos no sedimentables $[\mathrm{mg} / \mathrm{L}]$

$V_{0}$ : Velocidad máxima teórica de sedimentación $[\mathrm{dm} / \mathrm{d}]$

$V_{0}^{\prime}$ : Velocidad máxima práctica de sedimentación $[\mathrm{dm} / \mathrm{d}]$

$r_{h}$ : Parámetro característico de la sedimentación zonal [L/mg]

$r_{p}$ : Parámetro asociado al comportamiento de la velocidad de sedimentación a bajas concentraciones de sólidos [L/mg]

Para el modelado del sedimentador en este trabajo, se considera que el sedimentador está dividido en cinco capas de 0.2 L cada una y que la alimentación se produce en la capa intermedia. Las capas se consideran de igual volumen y diferente altura, debido a que en este caso el sedimentador es un cono de Imhoff. Debido a la que el sedimentador no posee una sección transversal constante, en las expresiones 
que describen la concentración de sólidos en cada capa se incluyen factores de corrección de forma, los cuales deben ser estimados en el proceso de optimización. En la estimación de parámetros en el sedimentador, además, deben estimarse los valores de los parámetros incluidos en la expresión (2).

\section{ESTIMACIÓN DE PARÁMETROS}

Para obtener un buen ajuste del modelo a los datos experimentales, es necesario realizar una estimación de los valores de varios parámetros del modelo. Primeramente, se realiza un análisis de sensibilidad, para determinar los parámetros que tienen una mayor influencia sobre la concentración de biomasa total. El análisis de sensibilidad se realiza en PROOSIS® utilizando el método de integración IDAS y los conceptos de clases y métodos. El análisis de sensibilidad en los reactores permitió establecer que el modelo tiene una elevada sensibilidad a las velocidades máximas específicas de crecimiento y a las constantes de inactivación de las microalgas y bacterias heterótrofas.

El planteamiento y la forma de resolver un problema de estimación de parámetros en términos de optimización considera que para cada valor del vector de parámetros $\boldsymbol{\theta}$ (variables de decisión) el modelo proporciona una predicción de la respuesta del sistema $\hat{y}(\boldsymbol{\theta})$ en un experimento determinado. Para esto es necesario tomar muestras de los datos de entradas $\mathbf{u}(\mathrm{t})$ y salidas $\mathbf{y}(\mathrm{t})$ del sistema real en un período de tiempo t. Al modelo se le aplica la misma secuencia de variables manipuladas que al sistema. Para cada instante de tiempo t, el error de predicción es un indicador de la bondad del modelo. En la figura 2 se representa el diagrama en bloques del estimador en el procedimiento de optimización secuencial.

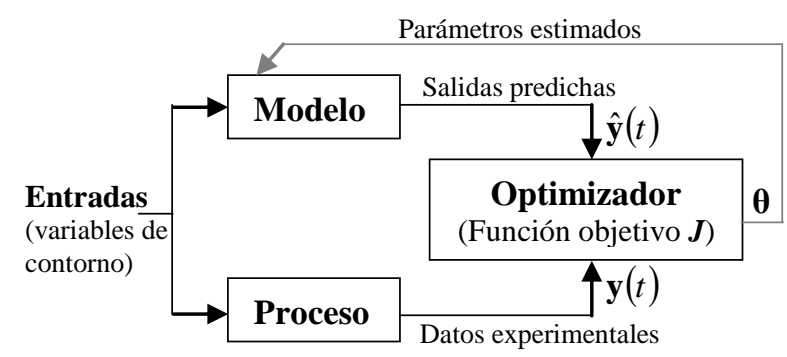

Figura 2. Estimador en la optimización secuencial

En el enfoque secuencial, se utiliza un simulador dinámico para resolver las ecuaciones diferenciales y calcular tanto la función de costo como las restricciones. Luego, un optimizador NLP (Nonlinear programming) se encarga de suministrar nuevos valores para las variables de decisión. El simulador recalcula y el ciclo se repite hasta que se alcanza cierto criterio de parada, normalmente que no se consiga mejorar significativamente la función de costo. En este trabajo se ha utilizado el algoritmo de programación no lineal SNOPT en el entorno de simulación dinámica PROOSIS ${ }^{\circledR}$ para resolver el problema de optimización. El método de integración seleccionado ha sido IDAS.

El estimador fair function se utiliza como función objetivo $J$ para la estimación de parámetros, debido a que esta, a diferencia de los estimadores basados en mínimos cuadrados, es robusta ante errores de medición que no presentan una distribución Gaussiana. El problema de optimización dinámica se plantea según (3):

$$
\min _{\hat{\theta}} J(\hat{\theta}, \theta)=\sum_{j \in M} c^{2}\left[\frac{\left|\varepsilon_{j}\right|}{c}-\log \left(1+\frac{\left|\varepsilon_{j}\right|}{c}\right)\right]
$$

Sujeto a las restricciones (4) y (5)

$$
\begin{gathered}
\frac{d \mathbf{x}(t)}{d t}=\mathbf{f}(\mathbf{x}(t), \mathbf{u}(t), \boldsymbol{\theta}, t) \\
\hat{\mathbf{y}}(t)=\mathbf{g}(\mathbf{x}(t), \mathbf{u}(t), \boldsymbol{\theta}, t) \\
\underline{\boldsymbol{\theta}} \leq \hat{\boldsymbol{\theta}} \leq \overline{\boldsymbol{\theta}}
\end{gathered}
$$

donde $\varepsilon_{j}=\left(\hat{\theta}_{j}-\theta_{j}\right) / \theta_{m}$ representa el error entre los datos experimentales $(\theta)$ y los estimados por el modelo $(\hat{\theta})$, limitados entre los límites mínimos y máximos definidos, $c \in \mathfrak{R}^{+}$es un parámetro de ajuste definido por el usuario y $\theta_{m}$ es el valor medio de los datos medidos. En la estimación de parámetros en ambos reactores y el sedimentador se elige $c=3$.

La estimación de parámetros en los reactores se realiza utilizando las variables de decisión seleccionadas en el análisis de sensibilidad y considerando como variables de salida del modelo el Total de Sólidos en Suspensión, el cual está dado por la suma de todos los componentes particulados del modelo (6).

$$
T S S=X_{A L G}+X_{H}+X_{A O B}+X_{N O B}+X_{I}+X_{S}
$$

En (6), $\mathrm{X}_{\mathrm{ALG}}$ representa la concentración de biomasa de algas, $X_{H}$ la concentración de bacterias heterótrofas, $\mathrm{X}_{\mathrm{AOB}}$ la concentración de bacterias oxidantes de amonio, $\mathrm{X}_{\mathrm{NOB}}$ la concentración de bacterias oxidantes de nitritos, $\mathrm{X}_{\mathrm{I}}$ la materia orgánica particulada inerte y $X_{S}$ la materia orgánica particulada lentamente biodegradable. En el modelo BIO_ALGAE las composiciones de los componentes particulados están expresadas en términos de Demanda Química de Oxígeno (Chemical Oxygen Demand, COD), por lo cual es necesario realizar la transformación de mgCOD/L a mgTSS/L para realizar la comparación con los datos experimentales. 
Para realizar este ajuste, se utilizaron las relaciones COD/TSS obtenidas en [5].

$$
T S S=\frac{X_{A L G}}{1.57}+\frac{\left(X_{H}+X_{A O B}+X_{N O B}+X_{I}+X_{S}\right)}{1.45}
$$

En la Tabla 1 se muestran los valores iniciales de los componentes utilizados para la estimación de parámetros del modelo en ambos reactores. Los valores iniciales de los componentes particulados se estimaron a partir del TSS, teniendo en cuenta las relaciones descritas en [15]. Los valores de $S_{I}$ y $S_{S}$ se determinaron a partir de los valores medidos de TOC disuelto.

Para la estimación de parámetros en el sedimentador, se consideran los valores medidos de TSS en el efluente clarificado y la corriente de purga. La expresión (3) se utiliza como función objetivo para la estimación de parámetros en el sedimentador. Se utilizan 11 variables de decisión.

En las tablas 2 y 3 se muestran los valores de las variables de decisión resultantes de estimación de parámetros en los reactores y el sedimentador, respectivamente. En ambos casos, los límites de las variables de decisión y los valores iniciales para la optimización se establecieron según los valores reportados en la literatura. Para la estimación de parámetros se utilizaron los datos experimentales correspondientes a los primeros 30 días de la etapa I, mientras que para la validación del modelo se utilizaron datos de la etapa I (días 31-47) y la etapa II (días 48-60).

Tabla 1: Concentraciones iniciales de los componentes en el reactor anóxico y el fotobiorreactor.

\begin{tabular}{|c|c|c|c|c|}
\hline Componentes & Descripción & Anóxico & Aerobio & Unidades \\
\hline $\mathrm{S}_{\mathrm{NH} 4}$ & Nitrógeno amónico & 54 & 60 & $\mathrm{mgN}-\mathrm{NH}_{4} \mathrm{~L}^{-1}$ \\
\hline $\mathrm{S}_{\mathrm{NH} 3}$ & Nitrógeno amoniacal & 3 & 5 & $\mathrm{mgN}-\mathrm{NH}_{3} \mathrm{~L}^{-1}$ \\
\hline $\mathrm{S}_{\mathrm{NO} 3}$ & Nitrato & 0.4 & 0 & $\mathrm{mgN}-\mathrm{NO}_{3} \mathrm{~L}^{-1}$ \\
\hline $\mathrm{S}_{\mathrm{NO} 2}$ & Nitrito & 0 & 0 & $\mathrm{mgN}-\mathrm{NO}_{2} \mathrm{~L}^{-1}$ \\
\hline $\mathrm{S}_{\mathrm{CO} 2}$ & Dióxido de carbono & 5 & 2 & $\mathrm{mgC}-\mathrm{CO}_{2} \mathrm{~L}^{-1}$ \\
\hline $\mathrm{S}_{\mathrm{HCO} 3}$ & Bicarbonato & 100 & 58 & $\mathrm{mgC}-\mathrm{HCO}_{3} \mathrm{~L}^{-1}$ \\
\hline $\mathrm{S}_{\mathrm{CO} 3}$ & Carbonato & 2 & 1 & $\mathrm{mgC}-\mathrm{CO}_{3} \mathrm{~L}^{-1}$ \\
\hline $\mathrm{S}_{\mathrm{PO} 4}$ & Fosfato & 903.8 & 797.9 & $\mathrm{mgP}-\mathrm{PO}_{4} \mathrm{~L}^{-1}$ \\
\hline $\mathrm{S}_{\mathrm{O} 2}$ & Oxígeno disuelto & 1 & 19.4 & $\mathrm{mgO}_{2} \mathrm{~L}^{-1}$ \\
\hline $\mathrm{S}_{\mathrm{H}}$ & Iones hidrógeno & $3.6 \mathrm{e}-08$ & $7.1 \mathrm{e}-09$ & $\mathrm{mgHL}^{-1}$ \\
\hline $\mathrm{S}_{\mathrm{OH}}$ & Iones hidróxido & $2.8 \mathrm{e}-07$ & $1.4 \mathrm{e}-06$ & $\mathrm{mgH}-\mathrm{OH} \mathrm{L}{ }^{-1}$ \\
\hline $\mathrm{S}_{\mathrm{S}}$ & Materia orgánica soluble rápidamente biodegradable & 191 & 169 & $\mathrm{mgCOD} \mathrm{L}^{-1}$ \\
\hline $\mathrm{S}_{\mathrm{I}}$ & Materia orgánica soluble inerte & 25.14 & 25.14 & $\mathrm{mgCOD} \mathrm{L}^{-1}$ \\
\hline $\mathrm{X}_{\mathrm{ALG}}$ & Microalgas & 1589 & 1845 & $\operatorname{mgTSS~L}{ }^{-1}$ \\
\hline $\mathrm{X}_{\mathrm{H}}$ & Bacterias heterótrofas & 813 & 944 & $\operatorname{mgTSS~L}{ }^{-1}$ \\
\hline $\mathrm{X}_{\mathrm{AOB}}$ & Bacterias oxidantes de amonio & 0 & 0 & $\operatorname{mgTSS~L}{ }^{-1}$ \\
\hline $\mathrm{X}_{\mathrm{NOB}}$ & Bacterias oxidantes de nitrito & 1.57 & 1.35 & $\operatorname{mgTSS~L}{ }^{-1}$ \\
\hline $\mathrm{X}_{\mathrm{S}}$ & Materia orgánica particulada lentamente biodegradable & 94.41 & 81.3 & $\operatorname{mgTSS~L}{ }^{-1}$ \\
\hline$X_{I}$ & Materia orgánica particulada inerte & 258.05 & 222.22 & $\operatorname{mgTSS~L}^{-1}$ \\
\hline
\end{tabular}

Tabla 2: Valores optimizados de los parámetros en ambos reactores (Modelo BIO_ALGAE)

\begin{tabular}{|l|l|l|}
\hline Parámetro & \multicolumn{1}{|c|}{ Descripción } & \multicolumn{1}{|c|}{ Valor } \\
\hline$\mu_{\mathrm{ALG}}$ & Velocidad máxima específica de crecimiento de microalgas & $0.4 \mathrm{~d}^{-1}$ \\
$\mu_{\mathrm{H}}$ & Velocidad máxima específica de crecimiento de bacterias heterótrofas & $1 \mathrm{~d}^{-1}$ \\
$\mathrm{k}_{\mathrm{death}, \mathrm{ALG}}$ & Constante de inactivación para microalgas & $0.21 \mathrm{~d}^{-1}$ \\
$\mathrm{k}_{\mathrm{death}, \mathrm{H}}$ & Constante de inactivación para bacterias heterótrofas & $0.9 \mathrm{~d}^{-1}$ \\
\hline
\end{tabular}

Tabla 3: Valores optimizados de los parámetros en el sedimentador

\begin{tabular}{|l|l|l|}
\hline Parámetro & \multicolumn{1}{|c|}{ Descripción } & \multicolumn{1}{|c|}{ Valor } \\
\hline$X_{\min }$ & Concentración de sólidos no sedimentables & $1 \mathrm{mg} / \mathrm{L}$ \\
$V_{0}$ & Velocidad máxima teórica de sedimentación & $6151.43 \mathrm{dm} / \mathrm{d}$ \\
$r_{h}$ & Parámetro característico de la sedimentación zonal & $0.0041 \mathrm{~L} / \mathrm{mg}$ \\
$r_{p}$ & Parámetro de velocidad de sedimentación a bajas concentraciones de TSS & $0.006 \mathrm{~L} / \mathrm{mg}$ \\
$a_{1}, a_{3}, b_{2}, b_{3}$ & Factores de corrección en el modelo & 1 \\
$a_{2}$ & Factor de corrección en el modelo & 0.1 \\
$b_{1}, c$ & Factores de corrección en el modelo & 1.6 \\
\hline
\end{tabular}




\section{RESULTADOS}

Utilizando los valores estimados de los parámetros, se comparan las salidas del modelo con los datos experimentales. En las figuras 3 y 4 se muestran los resultados del modelo para la predicción de la concentración total de biomasa en el reactor anóxico y el fotobiorreactor, respectivamente. Aunque se observan ciertas diferencias entre el modelo y los datos experimentales, estas se encuentran dentro de los límites permisibles. En el reactor anóxico, durante la etapa I, el valor medio de biomasa medida fue de $2575 \pm 160 \mathrm{mgTSS} / \mathrm{L}$ y el valor medio estimado fue de $2627 \pm 148 \mathrm{mgTSS} / \mathrm{L}$. Durante la etapa I en el fotobiorreactor se reportó un valor medio de TSS de $2531 \pm 191 \mathrm{mgTSS} / \mathrm{L}$; el valor medio estimado por el modelo fue de $2392 \pm 111 \mathrm{mgTSS} / \mathrm{L}$.

Las figuras 5 y 6 representan las salidas del modelo y los datos experimentales en el efluente clarificado y la purga del sedimentador, respectivamente. A pesar de apreciarse diferencias entre las salidas del modelo y los datos experimentales, los valores promedio del TSS resultan muy similares. Durante la etapa I, el valor medio del TSS de los datos experimentales en el efluente fue de $56 \pm 6 \mathrm{mgTSS} / \mathrm{L}$ (correspondiente con una eficiencia de eliminación de TSS del $98 \%$ ). El valor medio de TSS estimado por el modelo durante la etapa I fue de $55 \pm 5 \mathrm{mgTSS} / \mathrm{L}$ (eficiencia de eliminación de 97,7\%). Los valores experimentales y los calculados por el modelo se encuentran dentro de los límites permitidos por la legislación en cuanto a la concentración del TSS en el efluente y a la eficiencia de eliminación. Se observa la capacidad de predicción del modelo ante los cambios en el régimen de operación durante la etapa I (días 30-47) y la etapa II (días 47- 60).

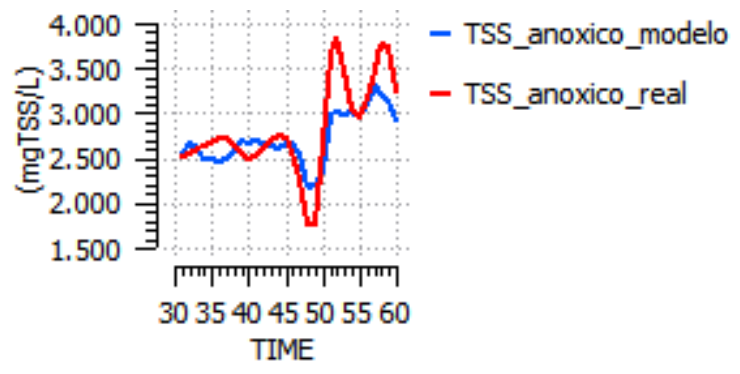

Figura 3. TSS en el reactor anóxico

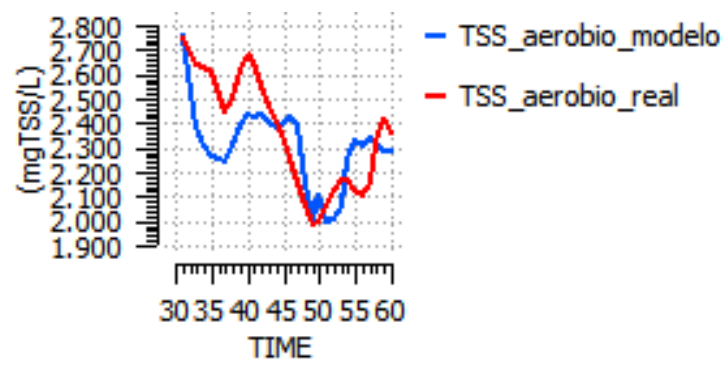

Figura 4. TSS en el fotobiorreactor aerobio

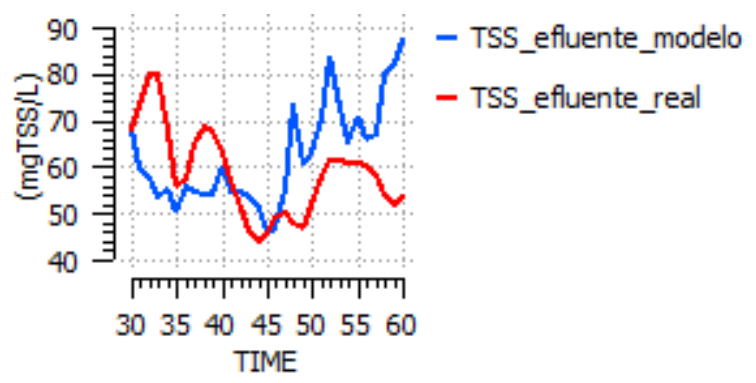

Figura 5. TSS en el efluente del sedimentador

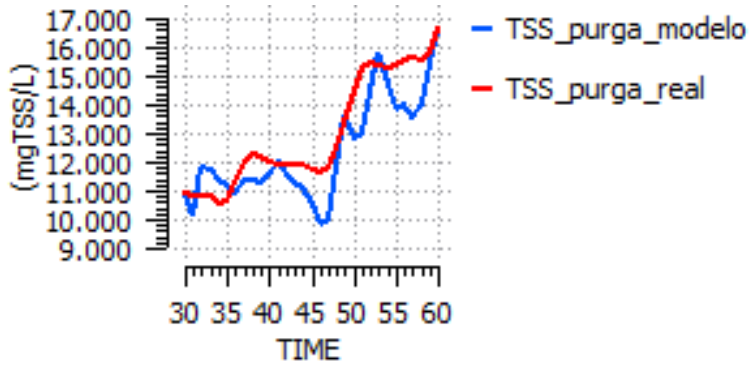

Figura 6. TSS en la purga del sedimentador

\section{CONCLUSIONES}

El modelo desarrollado permite estimar la concentración de biomasa en los reactores que conforman la instalación y en las corrientes de purga y efluente tratado. La estimación de parámetros ha permitido conseguir un buen ajuste del modelo a los datos experimentales.

Como trabajo futuro, se pretende ajustar el modelo a las otras salidas de las cuales se poseen datos experimentales. Esto permitirá mediante simulación, predecir la eficiencia de eliminación de nutrientes de la instalación y reducir significativamente el tiempo dedicado a la experimentación.

\section{Agradecimientos}

Este trabajo ha sido financiado por la Junta de Castilla y León y EU-FEDER (CLU 2017-09 y UIC 233) y por la Junta de Castilla y León y el Fondo Social Europeo (Orden EDU/601/2020).

BIOMASS ESTIMATION MODEL IN ANOXIC-AEROBIC ALGALBACTERIAL PHOTOBIOREACTOR CONFIGURATION FOR WASTEWATER TREATMENT

\author{
Abstract \\ This work deals with modelling of anoxic-aerobic \\ algal-bacterial photobioreactor with biomass
}


recycling for wastewater treatment. Total suspended solids concentration in elements composing installation is estimated through mathematical model. Parameter estimation in both reactors and settler is realized in order to fit model outputs to experimental data. Model validation is performed via simulation, comparing model outputs and experimental data.

Keywords: Algal-bacterial symbiosis, Modelling, Parameter estimation, Sedimentation, Wastewater treatment.

\section{Referencias}

[1] Acien, F., Fernández Sevilla, J., \& Molina Grima, E. (2013). Photobioreactors for the production of microalgae. Environ. Sci. Biotechnol., 12(2), 131-151.

[2] Alcántara, C., Domínguez, J., García, D., Blanco, S., Pérez, R., García-Encina, P., y otros. (2015). Evaluation of wastewater treatment in a novel anoxic-aerobic algal-bacterial photobioreactor with biomass recycling through carbon and nitrogen mass balances. Bioresource Technology, 191, 173-186.

[3] Arbib, Z., Ruiz, J., Álvarez-Díaz, P., GarridoPérez, C., Barragan, J., \& Perales, J. (2013). Effect of $\mathrm{pH}$ control by means of flue gas addition on three different photobioreactors treating urban wastewater in long-term Operation,. Ecol. Eng. , 57, 226-235.

[4] Brennan, L., \& Owende, P. (2010). Biofuels from microalgae-A review of technologies for production, processing, and extractions of biofuels and co-products. Renewable and Sustainable Energy Reviews, 14, 557-577.

[5] Casagli, F., Zuccaro, G., Bernard, O., Steyer, J.P., \& Ficara, E. (2021). ALBA: A comprehensive growth model to optimize algaebacteria wastewater treatment in raceway ponds. Water Research, 190(116734).

[6] CEE. Directiva 91/271/CEE SOBRE EL TRATAMIENTO DE LAS AGUAS RESIDUALES URBANAS. 1991.

[7] Chae, K., \& Kang, J. (2013). Estimating the energy independence of a municipal wastewater treatment plant incorporating green energy resources. Energy Convers. Manage., 72, 664672.
[8] De Godos, I., Vargas, V., Guzmán, H., Soto, R., García, B., García, P., y otros. (2014). Assessing carbon and nitrogen removal in a novel anoxic-aerobic cyanobacterial-bacterial photobioreactor configuration with enhanced biomass sedimentation. Water Res., 61, 77-85.

[9] "EcosimPro | PROOSIS. Modelling and Simulation Toolkits and Services" [Online]. Disponible en: www.ecosimpro.com

[10] EEE. (2013). VII Programa General de Acción de la Unión en Materia de Medio Ambiente hasta 2020 «Vivir bien, respetando los límites de nuestro planeta».

[11] Muñoz, R., \& Guieysse, B. (2006). Algalbacterial processes for the treatment of hazardous contaminants: a review. Water Res., 40, 2799-2815.

[12] Sánchez-Zurano, A., Rodríguez-Miranda, E., Guzmán, J., Acién-Fernández, F., FernándezSevilla, J., \& Molina Grima, E. (2021). ABACO: A New Model of Microalgae-Bacteria Consortia for Biological Treatment ofWastewaters. Appl. Sci., 11(998).

[13] Solimeno, A., \& García, J. (2017). Microalgaebacteria models evolution: From microalgae steady-state to integrated microalgae-bacteria wastewater treatment models- A comparative review. Science of the Total Environment, 607608, 1136-1150.

[14] Solimeno, A., Gómez-Serrano, C., \& Acién, F. G. (2019). BIO_ALGAE 2: improved model of microalgae and bacteria consortia for wastewater treatment. Environmental Science and Pollution Research , 26, 25855-25868.

[15] Solimeno, A., Parker, L., Lundquist, T., \& García, J. (2017). Integral microalgae-bacteria model (BIO_ALGAE): Application to wastewater high rate algal ponds. Science of the Total Environment, 601-602, 646-657.

[16] Takács, I., Nolasco, G., \& Patryioand, D. (1991). Clarification-Thickening Process. Water Research, 10(25), 1263-1271.

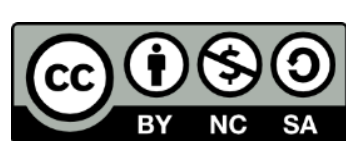
(C) 2021 by the authors. Submitted for possible open access publication under the terms and conditions of the Creative Commons Attribution CC BY-NC-SA $4.0 \quad$ license (https://creativecommons.org/licenses/byncsa/4.0/deed.es). 